Algebraic $8 \mathcal{G}$ Geometric $\mathcal{T}_{\text {opology }}$

Volume 4 (2004) 81-93

Published: 18 February 2004

ATG

\title{
A functorial approach to differential characters
}

\author{
PAul Turner
}

\begin{abstract}
We describe Cheeger-Simons differential characters in terms of a variant of Turaev's homotopy quantum field theories based on chains in a smooth manifold $X$.
\end{abstract}

AMS Classification 57R56; 53C05, 81T15, 58A10

Keywords Differential character, Homotopy quantum field theory

\section{Introduction}

Cheeger-Simons differential characters can be thought of equivalence classes of some "higher" version of line bundles-with-connection. In dimension two this can be taken to mean gerbes-with-connection, as explained in 2]. One way to think about "higher" line bundles-with-connection is in terms of Turaev's homotopy quantum field theories [9] (see also [8, 1]), where in dimension two such a thing provides a vector bundle over the free loop space together with a generalised (flat) connection where parallel transport is defined across surfaces. To make contact with gerbes and differential characters one needs to define a more rigid variation of 1+1-dimensional homotopy quantum field theory as explained [3] (see also [10] and for a similar approach [7]).

There is, however, an intrinsic difference between differential characters and homotopy quantum field theories. The former are defined in terms of homological information and the latter in terms of bordism. In dimension two this difference is unimportant (cf. the isomorphism between degree two homology and bordism) but in higher dimensions one would expect this difference to become apparent. The underlying geometrical picture of homotopy quantum field theories is however very appealing: one thinks of a bundle over some space of $n$-manifolds in $X$ with a generalised connection where parallel transport is defined across $(n+1)$-cobordisms. The motivation for the present work was to reconcile this picture with the homological needs of differential characters. 
The "functorial approach" of the title refers to the fact that many geometrical constructions can be defined in terms of representations of a geometrical category i.e. functors from a geometrical category to a category of vector spaces. Homotopy quantum field theories are a good example, but more familiar is the case of a line bundle-with-connection on $X$. One can define the path category of $X$ as the category with objects the points of $X$ and morphisms smooth paths between points. A line bundle with connection on $X$ can be thought of as a functor from the path category of $X$ to the category of one-dimensional vector spaces: a point in $X$ is assigned to its fibre and a path to parallel transport along that path. This functor must also be continuous in an appropriate way. This was the point of view in 3] where the authors gave similar description for gerbes-with-connection, by considering rank one representations of a category with objects loops in $X$ and morphisms equivalence classes of surfaces in $X$.

We recall now the definition of Cheeger-Simons differential characters [4. Letting $Z_{n+1} X$ denote the group of smooth $(n+1)$-cycles in $X$, a degree $n+1$ differential character is a homomorphism $f: Z_{n+1} X \rightarrow U(1)$ together with a closed $(n+2)$-form $c$ such that if $\beta$ is an $(n+2)$-chain then

$$
f(\partial \beta)=\exp \left(2 \pi i \int_{\beta} c\right) .
$$

The collection of these is denoted $\widehat{H}^{n+1}(X)$ where the index $n+1$ follows the convention in 4] (rather than that in [2] where the index $n+2$ is used for this group).

\section{Outline of the paper}

In order to marry the homological nature of differential characters with the functorial viewpoint we introduce new objects which we have dubbed chain field theories. These are symmetric monoidal functors from a category whose objects are smooth $n$-cycles in $X$ and whose morphisms are $(n+1)$-chains in $X$, to one-dimensional vector spaces. Such an object should be thought of as a line bundle over the group of $n$-cycles in $X$ together with a generalised connection in which parallel transport is defined across $(n+1)$-chains. The holonomy of such a bundle is a Cheeger-Simons differential character. The reader should beware that the bundle analogy only goes so far as we do not demand continuous functors (see also the remarks at the end of section 2). From one point of view, a chain field theory provides a possible interpretation of an $n$-gerbe-with-connection. 
In Section 1 we define the chain category of $X$, give the definition of chain field theory and give two important examples. In Section 2 we prove the following theorem.

Theorem 2.1 On a finite dimensional smooth manifold there is an isomorphism from the group of $(n+1)$-dimensional chain field theories (up to isomorphism) to the group of $(n+1)$-dimensional differential characters.

In Section 3 we characterise flat chain field theories as those that are invariant under deformation by $(n+2)$-chains and finally we discuss the classification of flat theories by the group $H^{n+1}(X ; U(1))$.

\section{Chain Field Theories}

We will construct a symmetric monoidal category, $\mathcal{G}_{n+1} X$ of $n$-cycles and $(n+$ 1)-chains in $X$, and then define a chain field theory to be a 1-dimensional representation of this category. Throughout we will work with cubical chains, for consistency with the work of Cheeger and Simons.

\section{Chain categories}

Let $X$ be a smooth finite dimensional manifold. Let $C_{k} X$ denote the group of smooth $k$-chains in $X$ and let $Z_{k} X$ (resp. $B_{k} X$ ) be the subgroup of smooth cycles (resp. boundaries).

The $(n+1)$-dimensional chain chain category of $X$, denoted $\mathcal{G}_{n+1} X$ is defined in the following way. The objects are smooth $n$-cycles in $X$ and a morphism from $\gamma$ to $\gamma^{\prime}$ is a smooth $(n+1)$-chain $\sigma$ satisfying $\partial \sigma=-\gamma+\gamma^{\prime}$. The composition $\sigma \circ \sigma^{\prime}$ is defined to be sum of chains $\sigma+\sigma^{\prime}$. Associativity follows from the fact that $C_{n+1} X$ is a group. Noting that the endomorphisms of an object $\gamma$ can be identified with the group of $(n+1)$-cycles, we take the zero cycle as the identity morphism for $\gamma$. To simplify notation we will write $\mathcal{G}$ for $\mathcal{G}_{n+1} X$ where there is no ambiguity and we will write $\mathcal{G}\left(\gamma, \gamma^{\prime}\right)$ for the set of morphisms from $\gamma$ to $\gamma^{\prime}$. We will also make no notational distinction between the identity morphisms for different $n$-cycles.

We define a bifunctor $\otimes: \mathcal{G} \times \mathcal{G} \rightarrow \mathcal{G}$ on objects by $\gamma_{1} \otimes \gamma_{2}=\gamma_{1}+\gamma_{2}$, where the sum on the right is taken in $Z_{n} X$ and on morphisms by $\sigma_{1} \otimes \sigma_{2}=\sigma_{1}+\sigma_{2}$, where the sum is taken in $C_{n+1} X$. This provides $\mathcal{G}$ with the structure of a monoidal category where the monoidal unit is the zero cycle in $Z_{n} X$. 
Proposition $1.1 \mathcal{G}$ is a strict symmetric strict monoidal groupoid. Its connected components are in one-to-one correspondence with $H_{n}(X ; \mathbb{Z})$.

Proof That $\mathcal{G}_{n+1} X$ is strict symmetric strict monoidal follows easily from the fact that $C_{n+1} X$ and $Z_{n} X$ and abelian groups.

To see that $\mathcal{G}$ is a groupoid, let $\sigma \in \mathcal{G}\left(\gamma, \gamma^{\prime}\right)$ and note that $-\sigma \in \mathcal{G}\left(\gamma^{\prime}, \gamma\right)$ since $\partial(-\sigma)=-\partial(\sigma)=-\left(-\gamma+\gamma^{\prime}\right)=-\gamma^{\prime}+\gamma$. Moreover $(-\sigma) \circ \sigma=\sigma+(-\sigma)=0$ which is the identity element in $\mathcal{G}(\gamma, \gamma)$.

To prove the statement about connected components observe that $\gamma$ is in the same path component as $\gamma^{\prime}$ if and only if there exists an $(n+1)$-chain $\sigma \in$ $\mathcal{G}\left(\gamma, \gamma^{\prime}\right)$ such that $\partial \sigma=-\gamma+\gamma^{\prime}$ i.e. $\gamma$ and $\gamma^{\prime}$ are homologous.

In fact, the objects of this category also possess inverses and $\mathcal{G}_{n+1} X$ is a categorical group i.e. a group object in the category of groupoids.

\section{The definition of chain field theories}

We let Lines denote the category with objects 1-dimensional complex vector spaces with Hermitian inner product and morphisms isometries. We regard this as a monoidal category under tensor product. For background information on monoidal categories, functors and so forth we refer to the appendix in 3 where all relevant definitions can be found.

An $(n+1)$-dimensional chain field theory on $X$ is a symmetric monoidal functor $E: \mathcal{G}_{n+1} X \rightarrow$ Lines together with a closed differential $(n+2)$-form $c$ such that for any $(n+2)$-chain $\beta$ the following holds:

$$
E(\partial \beta)(1)=\exp \left(2 \pi i \int_{\beta} c\right)
$$

The left hand side of this equation should be interpreted in the following manner. The boundary of an $(n+2)$-chain is an $(n+1)$-cycle and hence a morphism in $\mathcal{G}(0,0)$. Since 0 is the monoidal unit in $\mathcal{G}$ and the functor $E$ is monoidal there is an isomorphism $E(0) \cong \mathbb{C}$, and in this way $E(\partial \beta)$ is a unitary map $\mathbb{C} \rightarrow \mathbb{C}$. This condition should be thought of as a smoothness condition of the functor $E$. We note that as part of the definition of a monoidal functor there are natural isomorphisms $\Phi_{\gamma, \gamma^{\prime}}^{E}: E(\gamma) \otimes E\left(\gamma^{\prime}\right) \rightarrow E\left(\gamma+\gamma^{\prime}\right)$ for objects $\gamma$ and $\gamma^{\prime}$.

We say that a chain field theory is flat if the $(n+2)$-form $c$ is zero. The reader should think of a chain field theory as a line bundle over the space of 
$n$-cycles with parallel transport defined across $(n+1)$-chains. At first sight it is tempting to provide a more general definition in which the functor $E$ takes values in the category of hermitian vector spaces (rather than one-dimensional ones). However, the objects of $\mathcal{G}$ have inverses and $E$ is monoidal so for an object $\gamma$ we have $E(\gamma) \otimes E(-\gamma) \cong E(0) \cong \mathbb{C}$ from which it follows that $E(\gamma)$ is one dimensional.

Two chain field theories are isomorphic when there is a monoidal natural isomorphism between them. Recall that this requires a natural transformation $\Psi: E \rightarrow E^{\prime}$ such that for each object $\gamma$, the map $\Psi_{\gamma}: E(\gamma) \rightarrow E^{\prime}(\gamma)$ is an isomorphism and for each pair of objects $\gamma$ and $\gamma^{\prime}$

$$
\Psi_{\gamma+\gamma^{\prime}} \circ \Phi_{\gamma, \gamma^{\prime}}^{E}=\Phi_{\gamma, \gamma^{\prime}}^{E^{\prime}} \circ\left(\Psi_{\gamma} \otimes \Psi_{\gamma^{\prime}}\right) .
$$

The set of isomorphism classes of $(n+1)$-dimensional chain field theories on $X$ becomes a group, denoted $C h F T^{n+1}(X)$, with product $\star$ defined as follows. Given two theories $E$ and $F$ form $E \star F$ by defining $(E \star F)(\gamma)=E(\gamma) \otimes F(\gamma)$ and $(E \star F)(\sigma)=E(\sigma) \otimes F(\sigma)$. The $(n+2)$-form of $E \star F$ is the sum in the group of $(n+2)$-forms and the monoidal structure isomorphisms are the obvious ones. The identity of the group is the trivial chain field theory, which assigns all objects to $\mathbb{C}$ and all morphisms to the identity map. The $(n+2)$ form of the trivial chain field theory is the zero form and the monoidal structure isomorphisms are the canonical identification of $\mathbb{C} \otimes \mathbb{C}$ with $\mathbb{C}$. The inverse of $E$ is defined by $E^{-1}(\gamma)=E(\gamma)^{*}=\operatorname{Hom}(E(\gamma), \mathbb{C})$ and $E^{-1}(\sigma)=E(\sigma)^{*}$. The set of flat chain field theories forms a subgroup of this group.

A chain field theory has the following very useful invertibility property. Given a morphism $\sigma$ we have $E(-\sigma)=E(\sigma)^{-1}$. This is because

$E(-\sigma)=E(-\sigma) \circ E(\sigma) \circ E(\sigma)^{-1}=E(-\sigma+\sigma) \circ E(\sigma)^{-1}=E(0) \circ E(\sigma)^{-1}=E(\sigma)^{-1}$.

Just as line bundles with connection have holonomy defined for closed paths, a chain field theory has holonomy defined for closed $(n+1)$-chains i.e. $(n+1)$ cycles. If $\sigma$ is an $(n+1)$-cycle then it can be regarded as an element of $\mathcal{G}(0,0)$ and we define the holonomy of $\sigma$ by

$$
\mathrm{Hol}^{E}(\sigma)=E(\sigma)(1) .
$$

Notice that flat theories have trivial holonomy on boundaries since if $\beta$ is an $(n+2)$-chain then $\operatorname{Hol}^{E}(\partial \beta)=\exp \left(2 \pi i \int_{\beta} c\right)=1$.

If $\sigma \in \mathcal{G}(\gamma, \gamma)$ then $\partial \sigma=-\gamma+\gamma=0$ so we can also regard $\sigma$ as an element of $\mathcal{G}(0,0)$ and hence holonomy can be defined. As the next lemma shows, this holonomy is consistent with the map $E(\sigma): E(\gamma) \rightarrow E(\gamma)$. 
Lemma 1.2 If $\gamma$ is an object in $\mathcal{G}$ and $\sigma$ is an automorphism of $\gamma$, then the map $E(\sigma): E(\gamma) \rightarrow E(\gamma)$ is given by multiplication by $\operatorname{Hol}^{E}(\sigma)$.

Proof Since $E$ is a monoidal functor there is an isomorphism $\Phi: E(\gamma) \otimes$ $E(-\gamma) \cong E(0)=\mathbb{C}$. By naturality of the monoidal structure isomorphisms we have the following commutative diagram.

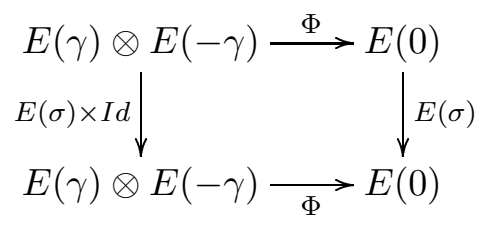

Letting $a$ and $b$ be generators of $E(\gamma)$ and $E(-\gamma)$ respectively we can write $E(\sigma)(a)=\lambda a$. By chasing $a \otimes b$ around the diagram one way we get $\Phi(a \otimes$ $b) \mathrm{Hol}^{E}(\sigma)$ and the other way $\lambda \Phi(a \otimes b)$. It follows that $\lambda=\operatorname{Hol}^{E}(\sigma)$.

This lemma has two corollaries which will be useful later on.

Corollary 1.3 If $\sigma_{1}$ and $\sigma_{2} \in \mathcal{G}\left(\gamma, \gamma^{\prime}\right)$ and $\operatorname{Hol}^{E}\left(\sigma_{1}-\sigma_{2}\right)=1$ then $E\left(\sigma_{1}\right)=$ $E\left(\sigma_{2}\right)$.

Proof We have that $E\left(-\sigma_{2}\right) \circ E\left(\sigma_{1}\right)=E\left(-\sigma_{2} \circ \sigma_{1}\right)=E\left(\sigma_{1}-\sigma_{2}\right)$ and using the lemma above we see that this is multiplication by $\operatorname{Hol}^{E}\left(\sigma_{1}-\sigma_{2}\right)=1$ i.e. $E\left(-\sigma_{2}\right) \circ E\left(\sigma_{1}\right)=I d_{E(\gamma)}$. Thus

$E\left(\sigma_{1}\right)=I d_{E\left(\gamma^{\prime}\right)} \circ E\left(\sigma_{1}\right)=E\left(\sigma_{2}\right) \circ E\left(-\sigma_{2}\right) \circ E\left(\sigma_{1}\right)=E\left(\sigma_{2}\right) \circ I d_{E(\gamma)}=E\left(\sigma_{2}\right)$.

Corollary 1.4 For a flat theory the holonomy of an $(n+1)$-cycle $\sigma$ depends only on the homology class $[\sigma] \in H_{n+1}(X, \mathbb{Z})$.

Proof Suppose $\sigma^{\prime}=\sigma+\partial \beta$ for some $(n+2)$-chain $\beta$. Then

$$
\operatorname{Hol}^{E}\left(\sigma^{\prime}\right) / \operatorname{Hol}^{E}(\sigma)=\operatorname{Hol}^{E}(-\sigma) \operatorname{Hol}^{E}\left(\sigma^{\prime}\right)=\operatorname{Hol}^{E}\left(-\sigma+\sigma^{\prime}\right)=\operatorname{Hol}^{E}(\partial \beta)=1 .
$$




\section{Examples}

We now give two of examples of chain field theories.

\section{Example 1.5}

In the first example we construct an $(n+1)$-dimensional chain field theory from an $(n+1)$-form. We let $\Omega^{*}(X)$ denote the smooth complex differential forms on $X$. By $\Omega^{*}(X)_{0, \mathbb{Z}}$ we denote the subspace of closed forms which have periods in $\mathbb{Z}$. Recall from [4] that there is an injection

$$
\Omega^{k}(X) \rightarrow \operatorname{Hom}\left(C_{k} X, U(1)\right)
$$

given by sending $\omega \in \Omega^{k}(X)$ to the map $\beta \mapsto \exp \left(2 \pi i \int_{\beta} \omega\right)$.

Let $\omega \in \Omega^{n+1}(X)$ and define a chain field theory $E^{\omega}: \mathcal{G}_{n+1} X \rightarrow$ Lines as follows. For any object $\gamma$ set $E^{\omega}(\gamma)=\mathbb{C}$ and for a morphism $(n+1)$-chain $\sigma$ define $E^{\omega}(\sigma): \mathbb{C} \rightarrow \mathbb{C}$ to be multiplication by $\exp \left(2 \pi i \int_{\sigma} \omega\right)$. The monoidal structure is the canonical one and the $(n+2)$-form $c$ is taken to be $d \omega$. Using Stokes theorem we see that for any $(n+2)$-chain $\beta$

$$
E^{\omega}(\partial \beta)(1)=\exp \left(2 \pi i \int_{\partial \beta} \omega\right)=\exp \left(2 \pi i \int_{\beta} c\right)
$$

as required.

As the differential on $(n+1)$-forms is linear this gives rise to a homomorphism

$$
\Omega^{n+1}(X) \rightarrow \operatorname{ChFT}^{n+1}(X) .
$$

Notice that if $\omega$ is closed then the chain field theory constructed above is flat. Moreover if two closed $(n+1)$-forms differ by an exact form then the resulting chain field theories are isomorphic. To see this let $\omega=\omega^{\prime}+d \theta$ for some $\theta \in \Omega^{n}(X)$. For an object $\gamma \in Z_{n} X$ define $\tau_{\gamma}: \mathbb{C}=E^{\omega}(\gamma) \rightarrow E^{\omega^{\prime}}(\gamma)=\mathbb{C}$ to be multiplication by $\exp \left(2 \pi i \int_{-\gamma} \theta\right)$. This defines a natural transformation $\tau: E^{\omega} \rightarrow E^{\omega^{\prime}}$. Thus (2) becomes a homomorphism

$$
H^{n+1}(X, U(1)) \rightarrow \text { Flat } C h F T^{n+1}(X) .
$$

\section{Example 1.6}

Now we construct a chain field theory from a Cheeger-Simons differential character. Recall (4] and 2]) that the Cheeger-Simons group of differential characters is defined by: 


$$
\begin{aligned}
\widehat{H}^{n+1}(X)=\left\{f \in \operatorname{Hom}\left(Z_{n+1} X, U(1)\right) \mid\right. & \exists c \in \Omega_{0, \mathbb{Z}}^{n+2}(X) \text { such that } \\
& \left.\forall \beta \in C_{n+2} X, f(\partial \beta)=\exp \left(2 \pi i \int_{\beta} c\right)\right\}
\end{aligned}
$$

This group fits in to the following exact sequences:

$$
\begin{aligned}
0 \rightarrow H^{n+1}(X, U(1)) & \rightarrow \widehat{H}^{n+1}(X) \stackrel{c}{\rightarrow} \Omega_{0, \mathbb{Z}}^{n+2}(X) \\
0 \rightarrow \Omega^{n+1}(X) / \Omega^{n+1}(X)_{0, \mathbb{Z}} & \rightarrow \widehat{H}^{n+1}(X) \rightarrow H^{n+2}(X, \mathbb{Z}) \rightarrow 0
\end{aligned}
$$

Starting with a differential character $f: Z_{n+1} X \rightarrow U(1)$ with $(n+2)$-form $c$ we will define an $(n+1)$-dimensional chain field theory $E^{f}: \mathcal{G} \rightarrow$ Lines as follows.

There is a short exact sequence

$$
0 \rightarrow Z_{n+1} X \stackrel{\iota}{\rightarrow} C_{n+1} X \stackrel{\partial}{\rightarrow} B_{n} X \rightarrow 0
$$

which gives rise to an exact sequence

$$
0 \rightarrow \operatorname{Hom}\left(B_{n} X, U(1)\right) \stackrel{\partial^{*}}{\rightarrow} \operatorname{Hom}\left(C_{n+1} X, U(1)\right) \stackrel{\iota^{*}}{\rightarrow} \operatorname{Hom}\left(Z_{n+1} X, U(1)\right) \rightarrow 0 .
$$

This sequence is exact on the left since $U(1)$ is divisible and it follows that $\operatorname{Ext}\left(Z_{n+1} X, U(1)\right)$ vanishes.

Using this exact sequence choose a lift $\tilde{f}: C_{n+1} X \rightarrow U(1)$ of $f$ and for objects set $E^{f}(\gamma)=\mathbb{C}$ and for morphisms define $E^{f}(\sigma): \mathbb{C} \rightarrow \mathbb{C}$ to be multiplication by $\tilde{f}(\sigma)$. The monoidal structure is taken to be the canonical one and the $(n+2)$-form is taken to be $c$.

That this provides a well defined symmetric monoidal functor follows from the fact that $C_{n+1} X$ is an abelian group. The condition on $c$ is also immediate since for any $\beta \in C_{n+2} X$ we have that $\partial \beta \in Z_{n+1} X$ so

$$
E(\partial \beta)(1)=\tilde{f}(\partial \beta)=f(\partial \beta)=\exp \left(2 \pi i \int_{\beta} c\right) .
$$

A priori this construction depends on the choice of lift of $f$, however another choice yields an isomorphic chain field theory. Moreover, the construction above is additive.

Proposition 1.7 The construction above provides a homomorphism of groups $\widehat{H}^{n+1}(X) \rightarrow \operatorname{ChFT}^{n+1}(X)$. 
Proof To show the construction is independent of the lift, let $\bar{f}$ be another lift which gives rise to another chain field theory $\bar{E}^{f}$ and claim that $E^{f}$ is isomorphic to $\bar{E}^{f}$.

Noting that $\tilde{f} / \bar{f} \in \operatorname{Ker}\left(\operatorname{Hom}\left(C_{n+1} X, U(1)\right) \rightarrow \operatorname{Hom}\left(Z_{n+1} X, U(1)\right)\right)$ by using the exact sequence (6) we can regard $\tilde{f} / \bar{f}$ as a homomorphism $B_{n} \rightarrow U(1)$. There is an exact sequence

$$
0 \rightarrow B_{n} X \rightarrow Z_{n} X \rightarrow H_{n}(X, \mathbb{Z}) \rightarrow 0
$$

and thus (again since $U(1)$ divisible) an exact sequence

$$
0 \rightarrow \operatorname{Hom}\left(H_{n}(X, \mathbb{Z}), U(1)\right) \rightarrow \operatorname{Hom}\left(Z_{n} X, U(1)\right) \rightarrow \operatorname{Hom}\left(B_{n} X, U(1)\right) \rightarrow 0 .
$$

Thus we can lift $\tilde{f} / \bar{f}$ to a homomorphism $h: Z_{n} X \rightarrow U(1)$. We now define a natural transformation $\tau: E^{f} \rightarrow \bar{E}^{f}$ as follows. For an object $n$-cycle $\gamma$ in $\mathcal{G}_{n+1} X$ define $\tau_{\gamma}: \mathbb{C}=E^{f}(\gamma) \rightarrow \bar{E}^{f}(\gamma)=\mathbb{C}$ to be multiplication by $h(\gamma)$. Note that since $h$ is a homomorphism $\tau$ satisfies $\tau_{\gamma+\gamma^{\prime}}=\tau_{\gamma} \tau_{\gamma^{\prime}}$ and $\tau_{-\gamma}=\tau_{\gamma}^{-1}$. To show that $\tau$ is natural we must show that for any morphism $\sigma$ from $\gamma$ to $\gamma^{\prime}$ we have $\tau_{\gamma}(1) \bar{f}(\sigma)=\tau_{\gamma^{\prime}}(1) \tilde{f}(\sigma)$. This is true since

$$
\bar{f}(\sigma) / \tilde{f}(\sigma)=(\bar{f} / \tilde{f})(\partial \sigma)=h\left(-\gamma+\gamma^{\prime}\right)=\tau_{-\gamma+\gamma^{\prime}}(1)=\tau_{-\gamma}(1) \tau_{\gamma^{\prime}}(1) .
$$

Thus, up to isomorphism, the construction above is independent of the choice of lift.

Finally, to see that we have a homomorphism we must show that for differential characters $f$ and $f^{\prime}$ we have an isomorphism $E^{f+f^{\prime}} \cong E^{f} \star E^{f^{\prime}}$. This follows immediately from the definition of $\star$ and the fact that if we have lifts $\tilde{f}$ and $\tilde{f}^{\prime}$ of $f$ and $f^{\prime}$ we can choose the lift of $f+f^{\prime}$ to be $\tilde{f}+\tilde{f}^{\prime}$, from which we see that the canonical identification of $\mathbb{C} \otimes \mathbb{C}$ with $\mathbb{C}$ provides an isomorphism from $E^{f} \star E^{f^{\prime}}$ to $E^{f+f^{\prime}}$.

If the $(n+2)$-form $c$ above is zero, then the chain field theory constructed above is flat and using exact sequence (4), we can regard the differential character as an element of $H^{n}(X, U(1))$ and there is a homomorphism

$$
H^{n+1}(X, U(1)) \rightarrow \text { FlatChFT } T^{n+1}(X) .
$$

This is the same homomorphism as (3). In fact Example 1.5 is a special case of Example 1.6. using the fact that an $n+1$-form $\omega$ determines a differential character by $f=\exp \left(2 \pi i \int \omega\right)$ and $c=d \omega$.

It is interesting to compare the example above with the constructions found in the integration theory of Freed and Quinn ( $6,[5])$ in the context of ChernSimons theory. 


\section{Classification by Cheeger-Simons groups}

We now show that equivalence classes of $(n+1)$-dimensional chain field theories are classified by the Cheeger-Simons group $\widehat{H}^{n+1}(X)$. Taking holonomy of a chain field theory defines a function

$$
\text { Hol: } \operatorname{ChFT}^{n+1}(X) \rightarrow \widehat{H}^{n+1}(X) .
$$

Recall the notation used before: the holonomy of $E$ is denoted $\mathrm{Hol}^{E}$. Using this notation the function $\mathrm{Hol}$ above sends $(E, c)$ to $\left(\mathrm{Hol}^{E}, c\right)$ and this function is a homomorphism of groups since

$$
\mathrm{Hol}^{E \star F}(\sigma)=(E \star F)(\sigma)(1)=E(\sigma)(1) F(\sigma)(1)=\mathrm{Hol}^{E}(\sigma) \mathrm{Hol}^{F}(\sigma) .
$$

The proof of the following theorem is a reformulation of the proof of the main theorem in [3].

Theorem 2.1 On a finite dimensional smooth manifold there is an isomorphism from the group of $(n+1)$-dimensional chain field theories (up to isomorphism) to the group of $(n+1)$-dimensional differential characters.

Proof We will show that the holonomy homomorphism Hol is an isomorphism with inverse provided by the homomorphism in Proposition 1.7.

Firstly, we will show that $\operatorname{Ker}(\mathrm{Hol})$ is trivial. Let $E \in \operatorname{Ker}(\mathrm{Hol})$, so $\operatorname{Hol}^{E}(\sigma)=1$ for all $\sigma \in Z_{n+1} X$. Writing $\underline{H}$ for the category with objects the elements of $H_{n}(X, \mathbb{Z})$ only identity morphisms we can assign to $E$ a symmetric monoidal functor $\underline{H} \rightarrow$ Lines as follows. Given objects $\gamma$ and $\gamma^{\prime}$ in the same connected component of $\mathcal{G}$ there is a canonical identification of $E(\gamma)$ with $E\left(\gamma^{\prime}\right)$, since if $\sigma_{1}$ and $\sigma_{2}$ are both morphisms from $\gamma$ to $\gamma^{\prime}$ then $\operatorname{Hol}^{E}\left(\sigma_{1}-\sigma_{2}\right)=1$ and hence by Corollary $1.3 E\left(\sigma_{1}\right)=E\left(\sigma_{2}\right)$. It follows from the fact that the connected components of $\mathcal{G}$ are in one-to-one correspondence with $H_{n}(X, \mathbb{Z})$ that we can associate a line $L_{x}$ to each $x \in H_{n}(X, \mathbb{Z})$. Since the morphisms in $\underline{H}$ are identities, this defines a functor $\underline{H} \rightarrow$ Lines. By choosing representatives for each $x \in H_{n}(X, \mathbb{Z})$, we can use the monoidal structure isomorphisms of $E$ to define natural isomorphisms $\Phi_{x, x^{\prime}}: L_{x} \otimes L_{x^{\prime}} \rightarrow L_{x+x^{\prime}}$ showing that the functor $\underline{H} \rightarrow$ Lines is monoidal and moreover symmetric.

Conversely given a symmetric monoidal functor $\phi: \underline{H} \rightarrow$ Lines we can construct a chain field theory with trivial holonomy by setting $E(\gamma)=\phi([\gamma])$ and $E(\sigma)=$ $I d$. This provides an identification of $\operatorname{Ker}(\mathrm{Hol})$ with the group of symmetric monoidal functors $\underline{H} \rightarrow$ Lines. Using Lemma 6.2 of $\underline{3}$ reformulated for $U(1)$ rather than $\mathbb{C}^{\times}$, the latter can be identified with $\operatorname{Ext}\left(H_{n}(X, \mathbb{Z}), U(1)\right)$, but this 
group is trivial since $U(1)$ is divisible. We have thus shown that the holonomy homomorphism is injective.

To see that Hol is surjective (and that the homomorphism in Proposition 1.7 provides an inverse) let $f$ be a differential character and claim that $\mathrm{Hol}^{E^{f}}=f$, where $E^{f}$ is the chain field theory produced in Proposition 1.7. This is immediate however, since for $\sigma \in Z_{n+1} X$ we know that $E^{f}(\sigma): \mathbb{C} \rightarrow \mathbb{C}$ is multiplication by $f(\sigma)$, so as an element of $U(1)$ we have $\operatorname{Hol}^{E^{f}}(\sigma)=E^{f}(\sigma)(1)=f(\sigma)$.

It is important to note that the theorem above relates equivalence classes of chain field theories with differential characters. If, for example, one chooses to interpret 1-dimensional characters as classifying equivalence classes of line bundles-with-connection then there is only an identification of line bundleswith-connection with chain field theories after quotienting up to equivalence. One could modify the definition of chain field theory so that the functor is continuous which would get closer to a genuinely geometric interpretation, but we haven't done that here. I am grateful to Simon Willerton and Mark Brightwell for clarifying this point.

\section{$3 \quad$ Flat theories}

In this section we show that flat chain field theories are characterised by invariance under deformation by $(n+2)$-chains. This is analogous to the fact that for flat line bundles parallel transport is invariant under deformation by homotopy.

Let $\sigma_{1}, \sigma_{2} \in \mathcal{G}\left(\gamma, \gamma^{\prime}\right)$ and suppose $\beta$ is an $(n+2)$-chain such that $\partial \beta=-\sigma_{1}+\sigma_{2}$. We say that a chain field theory $E$ is invariant under chain deformation if for all such $\sigma_{1}, \sigma_{2}$ and $\beta$ we have $E\left(\sigma_{1}\right)=E\left(\sigma_{2}\right)$.

Proposition 3.1 A chain field theory $E$ is flat if and only if it is invariant under chain deformation.

Proof We remarked after the definition of holonomy that if $E$ is flat then holonomy is trivial on boundaries. Thus

$$
\operatorname{Hol}^{E}\left(-\sigma_{1}+\sigma_{2}\right)=\operatorname{Hol}^{E}(\partial \beta)=1 .
$$

So by Corollary 1.3 , we see that $E\left(\sigma_{1}\right)=E\left(\sigma_{2}\right)$ and hence $E$ is invariant under chain deformation. 
Conversely, if $E$ is invariant under chain deformation we claim that $c=0$. Letting $\beta \in C_{n+1} X$ we can write $\partial \beta=-0+\partial \beta$ and so the definition of invariance under chain deformation implies $E(\partial \beta)=E(0)=I d$. Thus for all $\beta \in C_{n+2} X$

$$
\exp \left(2 \pi i \int_{\beta} c\right)=E(\partial \beta)(1)=1 .
$$

Using the injectivity of (1) we conclude that $c=0$.

This can be rephrased as follows. Define $\overline{\mathcal{G}}_{n+1}(X)$ to be the quotient category obtained from $\mathcal{G}_{n+1}(X)$ by imposing the following relation on morphisms. Let $\sigma_{1}, \sigma_{2} \in \mathcal{G}\left(\gamma, \gamma^{\prime}\right)$, then the relation is

$\sigma_{1} \sim \sigma_{2}$ iff there exists an $(n+2)$-chain $\beta$ such that $\partial \beta=-\sigma_{1}+\sigma_{2}$.

Composition is still well defined and the category inherits a monoidal structure from $\mathcal{G}_{n+1}(X)$.

The above proposition states that a flat chain field theory is one that factors through $\overline{\mathcal{G}}_{n+1}(X)$. Moreover it is clear that given a symmetric monoidal functor $\overline{\mathcal{G}}_{n+1}(X) \rightarrow$ Lines the composite $\mathcal{G}_{n+1}(X) \rightarrow \overline{\mathcal{G}}_{n+1}(X) \rightarrow$ Lines is a flat chain field theory and this assignment is one-to-one. Hence we have the following theorem.

Theorem 3.2 There is a one-to-one correspondence between flat chain field theories and symmetric monoidal functors $\overline{\mathcal{G}}_{n+1}(X) \rightarrow$ Lines.

Corollary 1.4 states that the holonomy of a flat chain field theory factors through $H_{n+1}(X, \mathbb{Z})$ and thus may be thought of as a homomorphism $H_{n+1}(X, \mathbb{Z}) \rightarrow$ $U(1)$. One may proceed as in the last section to study the function

$$
\text { Hol: Flat } C h F T^{n+1}(X) \rightarrow \operatorname{Hom}\left(H_{n+1}(X, \mathbb{Z}), U(1)\right) \cong H^{n+1}(X, U(1))
$$

to establish that this is an isomorphism of groups with the homomorphism (3) providing an inverse. As the proof is merely a reformulation of the proof of Theorem 2.1 and the result is expected once one knows that theorem (compare with the exact sequence (4)), we omit the details.

\section{Acknowledgement}

The author thanks Mark Brightwell, Marco Mackaay, Roger Picken and Simon Willerton for helpful comments. 


\section{References}

[1] M. Brightwell and P. Turner, Representations of the homotopy surface category of a simply connected space, J. Knot Theory Ramifications 9 (2000), no. 7, 855-864.

[2] J.-L. Brylinski, Loop spaces, characteristic classes and geometric quantization, Progr. Math., 107, Birkhäuser Boston, Boston, MA, 1993.

[3] U. Bunke, P. Turner and S. Willerton , Gerbes and homotopy quantum field theory, arXiv:math.AT/0201116

[4] J. Cheeger and J. Simons, Differential characters and geometric invariants, In LNM1167, pages 50-80. Springer Verlag, 1985.

[5] D. Freed, Higher algebraic structures and quantization, Comm. Math. Phys. 159 (1994), no. 2, 343-398.

[6] D. Freed and F. Quinn, Chern-Simons theory with finite gauge group, Comm. Math. Phys. 156 (1993), no. 3, 435-472.

[7] M. Mackaay and R. Picken, Holonomy and parallel transport for abelian gerbes, Adv. Math. 170, 287-339 (2002).

[8] G. Rodrigues, Homotopy quantum field theories and the homotopy cobordism category in dimension $1+1$, arXiv:math.QA/0105018.

[9] V. Turaev, Homotopy field theory in dimension 2 and group-algebras, arXiv: math.QA/9910010

[10] G. Segal, Topological structures in string theory, Topological methods in the physical sciences (London, 2000). R. Soc. Lond. Philos. Trans. Ser. A Math. Phys. Eng. Sci. 359 (2001), 1784, 1389-1398.

School of Mathematical and Computer Sciences

Heriot-Watt University

Edinburgh EH14 $4 A S$

Scotland

Email: paul@ma.hw.ac.uk

Received: 6 February 2003 